\title{
Changes in the state of paramagnetic centers and lattice parameter of micro-structured Si under the influence of weak magnetic field
}

\author{
V.V. Trachevsky ${ }^{1}$, L.P. Steblenko ${ }^{2}$, P.Y. Demchenko ${ }^{3}$, O.V. Koplak ${ }^{2}$, A.M. Kuryliuk ${ }^{2}$, A.K. Melnik ${ }^{1}$ \\ ${ }^{1}$ G.V. Kurdyumov Institute for Metal Physics, NAS of Ukraine, \\ 36, Vernadsky Ave., 03142 Kyiv, Ukraine \\ ${ }^{2}$ Physics Department, Taras Shevchenko Kyiv National University, \\ 2, Academician Glushkov Ave., 03680 Kyiv,_Ukraine; e-mail: koplak_o@ukr.net \\ ${ }^{3}$ I. Franko Lviv National University, Chemistry Department, \\ 6, Kyrylo and Mefodiy str., 79005 Lviv, Ukraine
}

\begin{abstract}
In this work, the influence of weak magnetic field on structure-dependent properties of micro-structured $\mathrm{Si}$ was determined. The researches of EPR-spectra inherent to micro-structured Si showed the presence of the spectral line at $H \sim 3500$ Oe that appears from centers with the $g$-factor $g \sim 2.0010$ ( $p_{в}-$ centers). Intensity of the determined spectral line decreases twice after magnetic processing. The observed redox processes and evolution of defect structure are interpreted as the influence of magnetic field on micro-structured Si. Calculations made using the data of X-ray diffractometric researches showed an essential decrease of internal strains and respective increase of the lattice parameter in micro-structured $\mathrm{Si}$ samples after magnetic processing in the weak magnetic field.
\end{abstract}

Keywords: silicon, structural defects, magnetic field, oxidation.

Manuscript received 18.10.09; accepted for publication 22.10.09; published online 30.12.09.

\section{Introduction}

It is known that physical properties of semiconductors are strongly determined by the state of subsystem structural defects. It is possible to get semiconductor materials with the determined physical-and-mechanical properties by introducing different defects into crystal and transforming their concentration hereby to have control over their distribution and interaction. Nowadays, on the one hand, the necessity in semiconductor materials used in various fields of science and technique, such as micro- and nano-electronics, is increasing, but on the other hand, requirements to structural perfection of functional semiconductors grow as well. In this connection, it seems topical both to practically use the structural defects inherent to semiconductors and to investigate the possibility of controlling them.

Recently, the ability to control physical properties of weakly-magnetic solid materials of various nature, in particular of semiconductor one, using the influence of weak magnetic fields caused researchers' interest [1-10]. It should be noted that the influence of weak magnetic fields on mechanical strength and plasticity, micromechanic, electro-physical and optical properties of weakly-magnetic crystals is out of the ordinary facts.
This phenomenon is reliably observed in experiments but does not belong to simply predictable physical facts. Its uncommonness is obvious: the energy of interaction inherent to diamagnetic crystal and its atomic components with magnetic field is extraordinary low. Therefore, its contribution to highly-energetic processes, particularly to those responsible for micro-mechanics, is scanty. So, the value of energy that can be transferred by such a magnetic field to a paramagnetic center is three times smaller than the heat energy $\mathrm{kT}$ at $300 \mathrm{~K}$. It is assumed that the existence of magnetic sensitive effects at such a low energy of magnetic interaction is related not with the energy of magnetic field itself but with the electron angular moment - spin, controlled by this magnetic field. Some researchers ground physical principles, according to which changes of properties of weakly magnetic crystals in weak magnetic field (MF) are based on spin conversion in point defects (structural nano-clusters), a consequence of which is weakening and break of chemical bonds [ $1-3]$. Thus, it is supposed that the role of MF is reduced to changes in the probability of spin-dependent transitions inside these nano-clusters as well as the probability of formation of intermediate spin configurations there, which corresponds to the possibility of formation of new nanoclusters with another atomic structure. However, up to 
date an ultimate conclusion about the nature of magnetostimulated effects in weakly-magnetic crystals, including the semiconductor ones, has not been done yet.

It should be mentioned that the specific weight of investigations devoted to studying the magnetodependent effects in $\mathrm{Si}$ crystals is relatively small as compared with other weakly-magnetic materials. The problem of changes in the state of paramagnetic centers in $\mathrm{Si}$, which are caused by magnetic field, is practically outfield. The problem of long-term relaxation of the lattice parameter after magnetic processing is also insufficiently explored.

Taking into account the above mentioned possibilities to use weak magnetic field for changing the real structure and physical properties of condensed matter, our work was aimed at studying the influence of this field on characteristics of paramagnetic centers, internal strains and lattice parameter in micro-structured silicon (MS).

\section{Experimental}

Si-powder used in the experiments as a sample of MS Si was obtained from residuals of $\mathrm{Si}$ milling. The experimental samples of $\mathrm{Si}$ after preparation were processed in magnetic field as follows: i) in the alternative electromagnetic low-frequency field with the induction $B=0.33$ T for 1 hour, ii) in the constant weak magnetic field with the induction $B=0.2 \mathrm{~T}$ for 60 days.

EPR spectra of paramagnetic centers were registered using EPR spectrometer E-580 (the frequency of pumping was $9.47 \mathrm{GHz}$, the frequency of modulation was $100 \mathrm{kHz}$ and the scanned range of $\mathrm{MF}$ was $\left.B_{0}=0.04-7 \mathrm{kOe}\right)$. When recording the spectra, we used double modulation of magnetic field and synchronous detection, which enabled us to register signals as the second derivative of electromagnetic emission absorption in the course of scanning this MF.

The lattice parameter and internal strains of MS Si were determined using X-ray diffraction study directly after magnetic influence and extraction of the samples from MF, as well as in regular intervals after magnetic processing. The massifs of experimental values for the intensity and diffraction angles for the samples were obtained using the automatic diffractometer STOE STADI P (produced by STOE \& Cie GmbH, Germany) with the linear position-sensitive detector PSD in accord to the Ginie scheme with modified geometry, the transmission method $\left(\mathrm{CuK} \alpha_{1}-\right.$ irradiation, bended $\mathrm{Ge}$ monochromator of the Iogann type; $2 \theta / \omega$-scan, the ambient temperature was $T=23.0 \pm 0.5^{\circ} \mathrm{C}, U=40 \mathrm{kV}$, $J=40 \mathrm{~mA}$ ).

\section{Results and discussion}

EPR spectra registered in the initial (standard) sample of MS $\mathrm{Si}$ and in the MS Si sample processed in the alternative electromagnetic low-frequency field are shown in Fig. 1.

Investigations of the EPR spectra of MS Si showed the availability of the spectral line at $H \sim 3500$ Oe that appears from the centers with the g-factor $g \sim 2.0010$. In Refs $[11,12]$ this spectral line is attributed to the socalled $p_{\mathrm{B}}$-centers. These centers are ascribed to destructed and broken Si bonds, on which the unpaired electron is localized. Paramagnetic $p_{B}$-centers are the major intrinsic defects inherent to the boundary $\mathrm{Si}_{-} \mathrm{SiO}_{2}$. Thus, the paramagnetic centers found in the studied samples are localized in the thin interface layer.

According to [11], the spin concentration strongly depends on the influence of different external factors. As one can see from the spectra given in Fig. 1, the maximal intensity of the observed spectral line that belongs to $\mathrm{p}_{\mathrm{B}}$-centers after $\mathrm{MP}$ is almost doubly decreased in the MS Si. This decrease in the intensity of EPR spectra indicates the decrease in the concentration of paramagnetic $\mathrm{p}_{\mathrm{B}}$-centers as a consequence of MP. The latter can be related with the following reasons. As it was mentioned in our previous papers $[13,14]$, the influence of MF on monocrystalline Si stimulates a spindependent process of destruction of chemical bonds, especially Si-O ones. As a result of this process that takes place during magnetic processing micro-structured $\mathrm{Si}$, the concentration of $\mathrm{Si}-\mathrm{O}$ bonds decreases to zero. Also, adsorption processes are related to the number of those initiated by magnetic influence [15]. The rate of these processes is increased due to growth of surface chemical activity as a consequence of magnetic processing. This processing enhances growing the thickness of natural surface oxidized layer, which we observed in monocrystalline $\mathrm{Si}$ by using X-ray photoelectronic spectroscopy. The thickness of this oxidized layer was four times increased after MP [16].

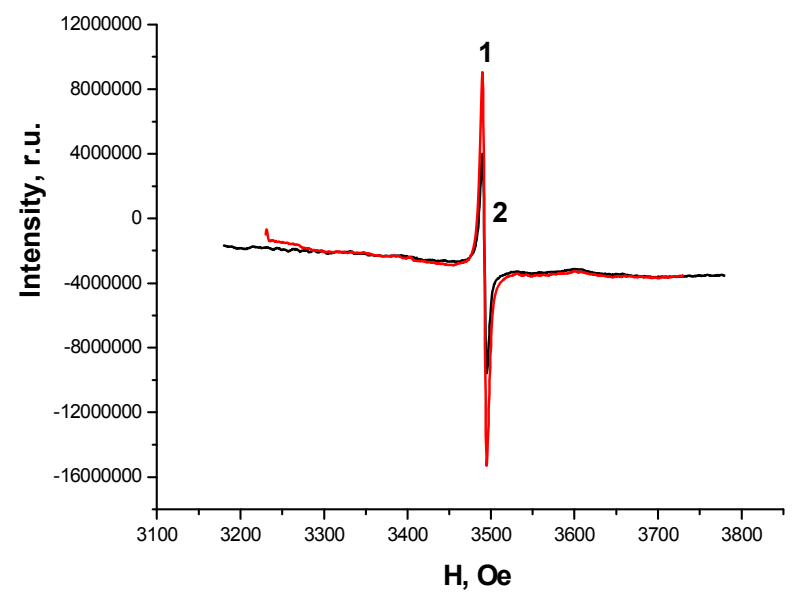

Fig. 1. EPR spectra registered in MS Si: 1 - sample without any magnetic processing; $2-$ sample processed with the alternative magnetic field. 
Thus, weak magnetic field stimulates not only distraction of $\mathrm{Si}-\mathrm{O}$ bonds, but also alternative redox process that takes place on monocrystalline surface in parallel.

It can be assumed that the influence of MF on MS $\mathrm{Si}$ could lead to redox processes similar to those observed by us in monocrystalline Si. The oxidizing-reducing reactions stimulated by $\mathrm{MF}$ in $\mathrm{MS} \mathrm{Si}$ could cause passivation of $\mathrm{Si}$ broken bonds at the boundary $\mathrm{Si}_{-} \mathrm{SiO}_{2} \quad\left(\mathrm{p}_{\mathrm{B}}\right.$-centers $)$ by adsorbed oxygen, which results in a decrease in the concentration of $p_{B}$ centers.

The observed data are indicative of the fact that the oxidation processes enhanced by MP on the surface of MS Si are rather intense, which is in a good accordance with the data of the investigation [17], where accelerated oxidation of micro-structured $\mathrm{Si}$ was revealed, as compared with that in bulk monocrystalline $\mathrm{Si}$.

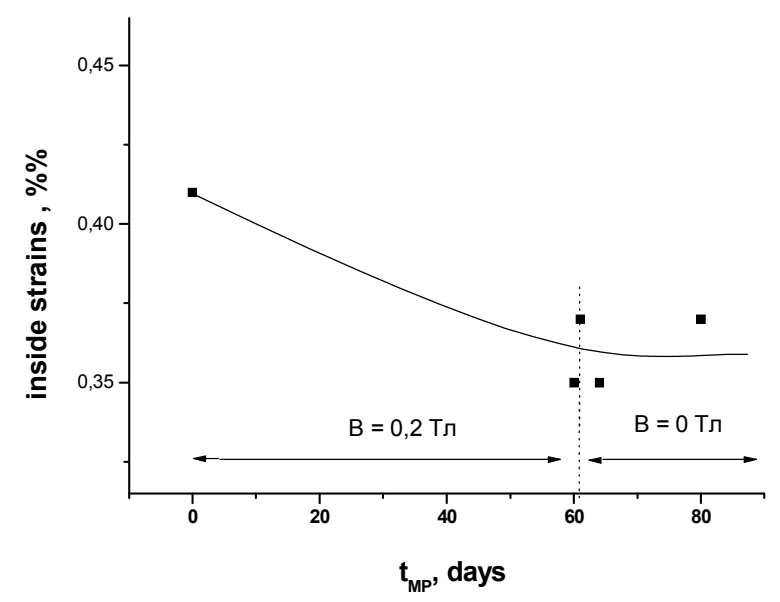

Fig. 2. Change of the internal strains in MS Si crystals stimulated by magnetic processing, duration of which was $t_{\mathrm{MP}}=60$ days.

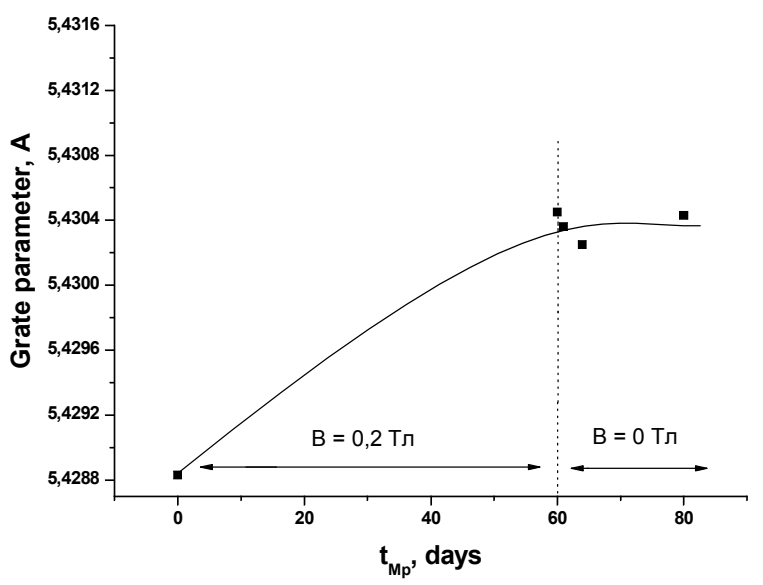

Fig. 3. Change of the lattice parameter in MS Si stimulated by magnetic processing, duration of which was $t_{\mathrm{MP}}=60$ days.

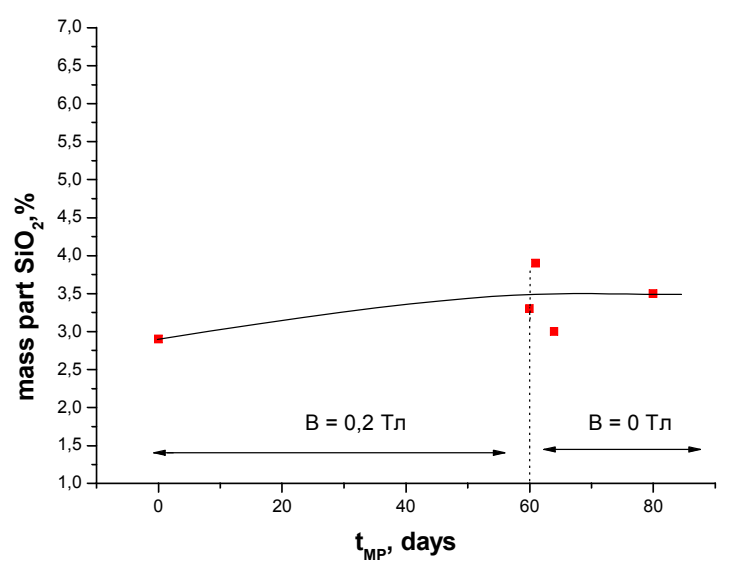

Fig. 4. Change in the mass fraction of oxidized phase in MS Si under the influence of magnetic processing. The induction of magnetic field $B=0.2 \mathrm{~T}$; duration of magnetic processing $t_{\mathrm{MP}}=$ 60 days.

It is probable that structural changes stimulated by MF at the sample surface should be reflected in changing the internal strains and lattice parameter. Really, as seen from the dependence depicted in Fig. 2, the internal strains in MS Si samples processed in the weak magnetic field were essentially decreased in the course of MP, as it was calculated using the X-ray diffractometric data. The decrease in internal strains corresponds to the increase of lattice parameter (Fig. 3).

In our opinion, the decrease of internal strains stimulated by magnetic processing and corresponding increase of the lattice parameter can be related with evolution of MS Si structure under the influence of MF. As seen from EPR data, this evolution lies in the essential decrease of the number of broken $\mathrm{Si}$ bonds after MP. It was already mentioned above that this decrease can be related with the MF ability to stimulate various processes including the redox ones connected with passivation of broken bonds. Lowering the number of broken Si bonds would decrease the internal strains and respective increase of the lattice parameter. We confirmed the availability of redox processes in microstructured $\mathrm{Si}$ after magnetic processing by using the Xray diffraction method. This method allowed determining the increase of the mass fraction for oxidized phase in micro-structured $\mathrm{Si}$ as a result of magnetic processing (Fig. 4).

As seen from Fig. 4, the oxide phase formed during the magnetic processing does not disappear even after its finish. Thus, the X-ray researches approved the results of investigation made using the EPR method, namely: the decrease in the concentration of broken bonds in microstructured $\mathrm{Si}$ is related with their passivation in the course of oxidizing-reducing reactions. 
References

1. M. Molotskii and V. Fleurov, Spin effect in plasticity // Phys. Rev. Lett. 78, p. 2779-2782 (1997).

2. M.I. Molotskii, Theoretical basis for electro- and magneto-plasticity // Mater. Sci. Eng. A 15(2), 288, p. 248-258 (2000).

3. A.L. Buchachenko, Physical kinetics of magnetoplasticity of diamagnetic crystals // Zhurnal eksperimental'noi $i$ teoreticheskoi fiziki 132(4), c. 827 - 830 (2007), in Russian.

4. M.N. Levin, B.A. Zon, Magnetic field induced generation of A-like centers in $\mathrm{Cz}-\mathrm{Si}$ crystals // Phys. Lett. A 260, p. 386-390 (1999).

5. R.B. Morgunov, Spin micromechanics in physics of plasticity // Uspekhi fizicheskikh nauk 174(2), p. 131-153 (2004), in Russian.

6. Yu.I. Golovin, R.B. Morgunov, A.A. Dmitrievskii, Influence of a weak magnetic field on spindependent relaxation of structural defects in diamagnetic crystals // Mater. Sci. Eng. A 15(2), Vol.288, p. 261-265 (2000).

7. G.I. Distler, V.M. Kanevskii, About influence of a weak magnetic field on real structure of solids // Doklady Akademii Nauk SSSR 268(3), p. 591 (1983), in Russian.

8. K.M. Salikhov, Yu. N. Molin, R.Z. Sagdeev and A.L. Buchachenko, Spin polarization and magnetic field effects in radical reactions, Yu.N. Molin (ed.). Elsevier, Amsterdam, 1984.

9. G.V. Semenova, T.P. Sushkova, V.V. Postnikov et al., Influence of pulsed magnetic fields on real structure of In-As crystals // Pis'ma $v$ zhurnal tekhnicheskoi fiziki 28(19), p. 50-55 (2002), in Russian.

10. M.N. Levin, V.N. Semenov, A.V. Naumov, Pulsed magnetic field processing of silicon substrates prior to thermal spray film deposition // Technical Physics Letters 27, p. 89-95 (1997).

11. A.B. Roitsin, V.M. Mayevskii, Electron paramagnetic resonance solid surface // Uspekhi fizicheskikh nauk 159(2), p. 297-333 (1998), in Russian.

12. V.S. Vavilov, V.F. Kiselev, B.N. Mukashev, Defects in Silicon and on its Surface. Moscow, Nauka Publ., 1990, p. 216 (in Russian).

13. V.A. Makara, M.A. Vasil'yev, L.P. Steblenko, O.V. Koplak, et al., Influence of magnetic field processing on microhardness and structure of nearsurface layers of silicon crystals // Fizyka i khimiya tverdogo tila 10(1), p. 193-199 (2009), in Ukrainian.

14. V.A. Makara, M.A. Vasil'yev, L.P. Steblenko, O.V. Koplak, et al., Changes of impurity composition and micro-hardness of silicon crystal subsurface layers as caused by magnetic field action // Fizika $i$ tekhnika poluprovodnikov 42(9), p. 1061-1064 (2008), in Russian.

15. M.N. Levin, A.V. Tatarintsev, O.A. Kostsov, A.M. Kostsov, Activation of semiconductor surface under influence of pulsed magnetic field // Zhurnal technicheskoi fiziki 73(10), p. 85-87 (2003), in Russian.

16. V.A. Makara, L.P. Steblenko, A.M. Korduban, O.V. Matveyeva, A.M. Kuryliuk et al., Change of physical-and-mechanical state and topology of surface of monocrystalline silicon under the influence of weak magnetostatic field // Proc. II Intern. Confer. "Materials and Structures of Modern Electronics” Minsk, Belorus', October 5-6, 2006, p. 189-192.

17. A.A. Kovalevskii, A.A. Shevchenok, A.S. Strogova, Peculiarities of oxidizing the micro- and nano-structured silicon powders // Neorganicheskie materialy 44(5), p. 519-523 (2008), in Russian. 